Editorial

\title{
Engineering Metamaterials: Present and Future
}

\author{
Stanislav Maslovski \\ Instituto de Telecomunicações and Departamento de Eletrónica, Telecomunicações e Informática, \\ Universidade de Aveiro, Campus Universitário de Santiago, 3810-193 Aveiro, Portugal; stas@av.it.pt
}

Received: 26 May 2020; Accepted: 29 May 2020; Published: 4 June 2020

\section{Introduction}

A couple of decades have passed since the advent of electromagnetic metamaterials. Although the research on artificial microwave materials dates back to the middle of the 20th century, the most prominent development in the electromagnetics of artificial media has happened in the new millennium. In the last two decades, the electromagnetics of one-, two-, and three-dimensional metamaterials acquired robust characterization, measurement, and design tools (e.g., [1-8]). Novel fabrication techniques have been developed. Many exotic effects involving metamaterials and metasurfaces, which initially belonged in a scientist's lab, are now well understood by practicing engineers. Therefore, when accepting the Guest Editor role for this Special Issue, I decided that it was the right moment to bring up and refresh the metamaterial concepts, which had to become a designer's tools of choice in the present-day electronics, microwaves, and photonics.

Time had shown that I made a step in the right direction. The papers published in this Special Issue had covered several important topics advancing the state of the art in telecommunications, subwavelength imaging, and biomedical sensing. Although some of these works build up on the metamaterial- and metasurface-related studies done in the last decade or even earlier, the originality in the selected papers resides in the approaches, models, and experimental techniques that demonstrate a great value for practical applications. That explains one of the reasons why this Special Issue has been entitled "Engineering Metamaterials". However, there is another reason: practical applications require from us, scientists and engineers, to search for metamaterial realizations that are compatible with the present-day technologies and respond to requests from foreseable future. That is why the engineers must be able to not just use, but also design and engineer metamaterials for a specific need. I believe, the reader will find this Special Issue useful for that purpose.

\section{The Present Issue}

The focus of this Special Issue has been on the theory and applications of electromagnetic metamaterials, metasurfaces, and metamaterial transmission lines as the building blocks of present-day and future electronic, photonic, and microwave devices. Below, I outline the main results obtained in the papers published in this Special Issue.

In Reference [9], the authors have studied the near-perfect tunneling in discrete metamaterial loaded waveguides. Electromagnetic wave tunneling in alternating $\varepsilon$-negative/ $\mu$-negative anisotropic metamaterial layers is studied with an ABCD-matrix-based method. A tunnel identification method is developed and demonstrated to reveal tunneling behavior experimentally. Two-dimensional imaging of permittivity distribution by an activated meta-structure with a functional scanning defect is studied in Reference [10]. A perforated metal plate with subwavelength holes and a needle-like conductor are employed to perform two-dimensional scanning. Imaging experiments have been conducted with the aim of detecting both conductive and dielectric samples for future biomedical applications concerning non-invasive and low-risk diagnosis. In Reference [11], analytical modeling of metamaterial differential transmission line using corrugated ground planes in high-speed printed circuit boards is performed. 
The developed model enables efficient and accurate prediction of the common-mode noise suppression and differential signal transmission characteristics. The authors of Reference [12] investigate wide-band dual polarization terahertz linear to circular polarization converters based on bi-layered transmissive metasurfaces. The bi-layered metasurfaces are formed by diagonally intersecting square metallic patches and rings. An equivalent circuit model is developed and validated with full-wave simulations. Reference [13] deals with a dual-band compact metamaterial absorber with fractal geometry. The used fractal structure allows for creating a dual-band metamaterial absorber with reduced unit cell size and small substrate thickness. Based on this principle, an absorber panel has been experimentally realized and validated. In Reference [14], a microfluidic sensor based on a composite left-right handed transmission line has been proposed and investigated. A change in the properties of the fluid that fills the microfluidic reservoir causes a change in the effective substrate permittivity, which subsequently changes the phase velocity in the transmission line. The studied sensor is characterized by relatively high sensitivity and good linearity and can be used for the biomass estimation inside microfluidic bioreactors. Two-bit terahertz encoder realized by graphene-based metamaterials is proposed and studied in Reference [15]. The encoder involves graphene-based metamaterials, in which the graphene structures are controlled by electric voltage applied to external electrodes. The authors foresee that their encoder can promote the development of multifunctional and integrated devices for future THz-band communications.

\section{Future}

Rapid growth in telecommunications and electronics requires development of new and original metamaterial-based and (or) metamaterial-inspired devices. Recently, digital metamaterials and programmable metasurfaces have attracted a lot of attention. Programmable metasurfaces are versatile tools for controlling electromagnetic wave propagation and performing almost instantaneous operations on the wavefronts of passing electromagnetic waves. It is without doubt that the metamaterial concepts (including the ones presented in this Special Issue) will be further developed for such applications.

Telecommunications in the mm-wave and $\mathrm{THz}$ bands will bring new challenges that will be addressed, in particular, by making use of materials with exotic electronic properties such as the graphene. In the last few years, topological metamaterials and effects have added an entirely new dimension to the whole picture. Emerging artificial intelligence techniques already assist researchers in design of novel metamaterials and devices based on them. Overall, I believe that there is a bright future for these new technologies and their applications in engineering metamaterials.

Acknowledgments: Here, first of all, I would like to thank all the Special Issue authors for their valuable contributions and acknowledge the work of anonymous reviewers and the editorial board of Electronics. Without your effort this Special Issue could not become a reality! The work related to Guest Editing of this Special Issue and publication of this Editorial was funded by FCT/MCTES, Portugal, through national funds and when applicable co-funded by EU funds under the project UIDB/50008/2020-UIDP/50008/2020.

Conflicts of Interest: The author declares no conflict of interest.

\section{References}

1. Tretyakov, S.A.; Nefedov, I.S.; Simovski, C.R.; Maslovski, S.I. Modelling and Microwave Properties of Artificial Materials with Negative Parameters. In Advances in Electromagnetics of Complex Media and Metamaterials, NATO Science Series (Series II: Mathematics, Physics and Chemistry); Zouhdi, S., Sihvola, A., Arsalane, M., Eds.; Springer: Dordrecht, The Netherlands, 2003; Chapter 89, pp. 99-122. [CrossRef]

2. Kärkkäinen, M.K.; Maslovski, S.I. Wave propagation, refraction, and focusing phenomena in Lorentzian double-negative materials: A theoretical and numerical study. Microw. Opt. Technol. Lett. 2003, 37, 4-7. [CrossRef]

3. Maslovski, S.; Tretyakov, S.; Alitalo, P. Near-field enhancement and imaging in double planar polariton-resonant structures. J. Appl. Phys. 2004, 96, 1293-1300. [CrossRef] 
4. Alitalo, P.; Maslovski, S.; Tretyakov, S. Near-field enhancement and imaging in double cylindrical polariton-resonant structures: Enlarging superlens. Phys. Lett. A 2006, 357, 397-400. [CrossRef]

5. Maslovski, S.I.; Silveirinha, M.G. Nonlocal permittivity from a quasistatic model for a class of wire media. Phys. Rev. B 2009, 80, 245101. [CrossRef]

6. Costa, J.T.; Silveirinha, M.G.; Maslovski, S.I. Finite-difference frequency-domain method for the extraction of effective parameters of metamaterials. Phys. Rev. B 2009, 80, 235124. [CrossRef]

7. Maslovski, S.I.; Morgado, T.A.; Silveirinha, M.G.; Kaipa, C.S.R.; Yakovlev, A.B. Generalized additional boundary conditions for wire media. New J. Phys. 2010, 12, 113047. [CrossRef]

8. Luukkonen, O.; Maslovski, S.I.; Tretyakov, S.A. A Stepwise Nicolson-Ross-Weir-Based Material Parameter Extraction Method. IEEE Antennas Wirel. Propag. Lett. 2011, 10, 1295-1298. [CrossRef]

9. Eccleston, K.W.; Platt, I.G. Identifying Near-Perfect Tunneling in Discrete Metamaterial Loaded Waveguides. Electronics 2019, 8, 84. [CrossRef]

10. Itami, G.; Sakai, O.; Harada, Y. Two-Dimensional Imaging of Permittivity Distribution by an Activated Meta-Structure with a Functional Scanning Defect. Electronics 2019, 8, 239. [CrossRef]

11. Kim, M. Analytical Modeling of Metamaterial Differential Transmission Line Using Corrugated Ground Planes in High-Speed Printed Circuit Boards. Electronics 2019, 8, 299. [CrossRef]

12. Fahad, A.K.; Ruan, C.; Chen, K. Dual-Wide-Band Dual Polarization Terahertz Linear to Circular Polarization Converters based on Bi-Layered Transmissive Metasurfaces. Electronics 2019, 8, 869. [CrossRef]

13. Venneri, F.; Costanzo, S.; Borgia, A. A Dual-Band Compact Metamaterial Absorber with Fractal Geometry. Electronics 2019, 8, 879. [CrossRef]

14. Radonić, V.; Birgermajer, S.; Podunavac, I.; Djisalov, M.; Gadjanski, I.; Kitić, G. Microfluidic Sensor Based on Composite Left-Right Handed Transmission Line. Electronics 2019, 8, 1475. [CrossRef]

15. Yin, S.; Shi, X.; Huang, W.; Zhang, W.; Hu, F.; Qin, Z.; Xiong, X. Two-Bit Terahertz Encoder Realized by Graphene-Based Metamaterials. Electronics 2019, 8, 1528. [CrossRef]

(C) 2020 by the author. Licensee MDPI, Basel, Switzerland. This article is an open access article distributed under the terms and conditions of the Creative Commons Attribution (CC BY) license (http://creativecommons.org/licenses/by/4.0/). 